\title{
BMJ Open Multidimensional assessment of women after severe maternal morbidity: the COMMAG cohort study
}

Elton C Ferreira, ${ }^{1}$ Maria Laura Costa, ${ }^{1}$ Rodolfo C Pacagnella, ${ }^{1}$ Carla Silveira, ${ }^{1}$ Carla B Andreucci, ${ }^{1}$ Dulce Maria Toledo Zanardi, ${ }^{1}$ Juliana P Santos, ${ }^{1}$ Carina R Angelini, ${ }^{1}$ Renato T Souza, ${ }^{1}$ Mary A Parpinelli, ${ }^{1}$ Maria Helena Sousa, ${ }^{2}$ Jose Guilherme Cecatti (D) ${ }^{1}$

To cite: Ferreira EC, Costa ML, Pacagnella RC, et al. Multidimensional assessment of women after severe maternal morbidity: the COMMAG cohort study. BMJ Open 2020;10:e041138. doi:10.1136/ bmjopen-2020-041138

- Prepublication history for this paper is available online. To view these files, please visit the journal online (http://dx.doi. org/10.1136/bmjopen-2020041138).

Received 31 May 2020 Revised 19 0ctober 2020 Accepted 15 November 2020

Check for updates

(c) Author(s) (or their employer(s)) 2020. Re-use permitted under CC BY-NC. No commercial re-use. See rights and permissions. Published by BMJ.

${ }^{1}$ Department of Obstetrics and Gynecology, State University of Campinas, Campinas, Brazil ${ }^{2}$ Department of Statistics, Faculdade de Medicina de Jundiai, Jundiai, Brazil

Correspondence to Professor Jose Guilherme Cecatti; cecatti@unicamp.br

\section{ABSTRACT}

Objectives To perform a multidimensional assessment of women who experienced severe maternal morbidity (SMM) and its short-term and medium-term impact on the lives and health of women and their children.

Design A retrospective cohort study.

Setting A tertiary maternity hospital from the southeast region of Brazil.

Participants The exposed population was selected from intensive care unit admissions if presenting any diagnostic criteria for SMM. Controls were randomly selected among women without SMM admitted to the same maternity and same time of childbirth.

Primary and secondary outcome variables Validated tools were applied, addressing post-traumatic stress disorder (PTSD) and quality of life (SF-36) by phone, and then general and reproductive health, functioning (WHO Disability Assessment Schedule), sexual function (Female Sexual Function Index (FSFI)), substance abuse (Alcohol, Smoking and Substance Involvement Screening Test 2.0) and growth/development (Denver Developmental Screening Test) of children born in the index pregnancy in a face-to-face interview.

Results All instruments were applied to 638 women ( 315 had SMM; 323 were controls, with the assessment of 264 and 307 children, respectively). SF-36 score was significantly lower in the SMM group, while PTSD score was similar between groups. Women who had SMM became more frequently sterile, had more abnormal clinical conditions after the index pregnancy and a higher score for altered functioning, while proportions of FSFI score or any drug use were similar between groups. Furthermore, children from the SMM group were more likely to have weight (threefold) and height (1.5 fold) for age deficits and also impaired development (1.5-fold). Conclusion SMM impairs some aspects of the lives of women and their children. The focus should be directed towards monitoring these women and their children after birth, ensuring accessibility to health services and reducing short-term and medium-term repercussions on physical, reproductive and psychosocial health.

\section{INTRODUCTION}

In the last few years, considerable effort has been made to define and classify maternal
Strengths and limitations of this study

The retrospective design of this cohort study is one of its limitations.

- As a retrospective cohort, it was not possible to better assess the conditions associated with death among the apparent excess cases in the exposed group.

- The compliance with the face-to-face interview was $76 \%$ (638 among 840 eligible women).

- This is the most complete assessment of consequences for the women surviving an experience of severe maternal morbidity with a multidimensional approach.

- The current study is the first to evaluate the consequences of severe maternal morbidity also for the children.

morbidity, ${ }^{1-3}$ due to a clear understanding that maternal deaths only represent a fraction of the problem and that it is possible to have a better knowledge of the burden of this disease exploring the extent of severe morbidity and near miss cases. ${ }^{45}$

The WHO defines maternal 'near miss' $(\mathrm{MNM})$ as women surviving a severe clinical condition during pregnancy, delivery or in the first 42 days postpartum, according to the specific laboratory, clinical or management criteria that consolidate organ dysfunction or failure. Potentially life-threatening conditions (PLTCs) are also considered and involve the occurrence of less severe conditions. Both conditions are defined as severe maternal morbidity (SMM). ${ }^{6}$ The higher number of cases and the opportunity to directly interview survivors of severe complications are fundamental to allow interventions that improve maternal health. In the last decade, several consistent studies were aimed at describing the local and global prevalence of near miss events. ${ }^{3}$ 
There is a growing understanding that severe obstetric complications may lead to long-term adverse conditions. ${ }^{7-9}$ However, few studies have evaluated the impact of maternal morbidity on the life of a woman after childbirth. Most studies had a very short follow-up or an insufficient number of cases. There is an increased rate of hypertensive disorders after a 1-year follow-up, ${ }^{8}$ reductions in future reproductive potential and also increased risk of death and complications in subsequent pregnancies, ${ }^{9}$ in addition to some impairment in sexual function. ${ }^{10}$

Therefore, the current study entitled COMMAG (Brazilian Severe Maternal Morbidity Cohort Study) ${ }^{511}$ was designed to perform a multidimensional assessment of women who experienced an SMM episode, compared with those without any severe morbidity. The aim of the current manuscript is to consolidate the results of all tools to provide a general, comprehensive and multidimensional overview of the medium-term and long-term impact of SMM on woman's health and life. Also, it was planned to check whether SMM affects one or several aspects simultaneously. It seeks to assess the burden on the lives of women, including reproductive health, quality of life, post-traumatic stress disorder, female sexual function, daily functioning, substance abuse and the physical, neurological and psychomotor development of children born after a complicated pregnancy. The purpose is to understand if the impact of SMM is captured in the same manner by different tools. A more simplified and effective evaluation of women could potentially occur in the postpartum period.

\section{METHODS}

\section{Study design and setting}

This was a retrospective cohort study performed in the maternity of the University of Campinas, Brazil, a tertiary public referral hospital for high-risk pregnancies. Analysis of the general and reproductive health of women who had given birth in this institution from 1 July 2008 to 30 June 2012 was conducted, in addition to other conditions, in a study entitled Cohort for Severe Maternal Morbidity (COMMAG) - multidimensional assessment of long-term repercussions of severe maternal morbidity already described in detail in previous publications. ${ }^{5} 11$ In 2013, the eligible women were traced to be invited to participate, therefore after 6 months -5 years of the index pregnancy.

\section{Sample size estimation}

The sample size was estimated for the entire cohort, considering all outcomes, especially the evaluation of disability and functioning (WHO Disability Assessment Schedule (WHODAS) 2.0). A total of 420 women for each group was considered sufficient for the assessment of outcomes covered on the telephone component. Estimating a 25\% loss of women accepting and attending a face-to-face interview component, this resulted in 315 women per group. Studies addressing the possible influences of SMM on short-term or long-term maternal consequences applying the new WHO definition and criteria are still lacking. Therefore, this estimated sample size was considered enough to provide power for all aspects included in the cohort study.

\section{Participants}

Briefly, to include women in the SMM group, researchers obtained retrospective data from medical charts of intensive care unit admissions and selected women who had presented some of the defined diagnostic criteria for SMM (PLTC or MNM). ${ }^{6}$ All women so classified during the study period were traced for being invited to participate in the study. A random sample of women who had given birth in the same maternity at the same time and had not developed SMM was enrolled as controls at a ratio of $1: 1$.

\section{Outcome variables and procedures}

Standard tools were applied to both groups of women and their respective children, exploring aspects of general and reproductive health, quality of life (SF-36) ${ }^{12}$ post-traumatic stress disorder (PTSD),${ }^{13}$ sexual function (Female Sexual Function Index (FSFI)), ${ }^{14}$ functioning and disabilities (WHODAS 2.0) ${ }^{15}$ use of licit and illicit substances (Alcohol, Smoking and Substance Involvement Screening Test 2.0 (ASSIST) $)^{16}$ and growth and development of children (weight and height for age and Denver Developmental Screening Test). ${ }^{17}$ Assessment occurred at different time points after childbirth, between 6 months and 5 years. Detailed analyses specifically for these instruments have already been performed and published. ${ }^{18-22}$

Eligible women were traced and invited by telephone to participate in the study. Those who agreed had the informed consent term read by trained interviewers, and their acceptance was further recorded. An initial interview was performed immediately by telephone, using computer-assisted telephone interview ${ }^{23}$ for two instruments-SF-36 (quality of life) ${ }^{12}$ and PTSD. ${ }^{13}$ At the end of the telephone interview, the participants were invited to take part in the second part of the study assessment, a face-to-face interview also including child health assessment, scheduled according to the availability of the participant, with a refund of costs.

For the second part of the study, researchers applied the other instruments after a new informed consent form was read and signed. These tools included information on general and reproductive health in a specific form developed specifically for this study as well as the remaining instruments.

The Medical Outcomes Study 36-item short form (SF-36) health survey is a multidimensional questionnaire, consisting of 36 items covering eight dimensions: physical functioning, social functioning, role limitation related to physical problems, role limitation related to emotional problems, pain, mental health, vitality and general health perception. The final score ranges from 
0 to 100 , where 0 is the worst general health status and a score of 100 corresponds to the best overall health. ${ }^{12}$

PTSD was evaluated by the PTSD Checklist-Civilian version, composed of 17 questions, with a severity scale ranging from 1 to 5 (nothing to much) for each question. ${ }^{13}$ Five questions refer to resurgence symptoms, seven concerned avoidance/emotional numbing and five were related to hyperexcitability symptoms. Higher scores are directly related to worse outcomes. ${ }^{13}$ Scores of 50 or higher were considered altered.

The FSFI questionnaire is a practical tool to evaluate the different strengths of each sexual function domain, transforming subjective into objective measures. It has already been applied to populations with multiple dysfunctions. A cut-off point for the diagnosis or suspected sexual dysfunction can be used. ${ }^{24}$

The 36-item WHODAS 2.0 version intends to measure activity functioning and participation in daily living activities in the last 30 days. It includes six domains: cognition (evaluates communication and thinking activities, including concentration, memory, problem solving, learning and communication), mobility (evaluates activities such as standing up, moving around inside the house, going outside the house and walking a long distance), self-care (evaluates hygiene, getting dressed, eating and staying alone), relationship with people (evaluates interaction with others and difficulties that may be encountered due to adverse health conditions), life activities (evaluates difficulty with daily living activities: household responsibilities, leisure, work and school); and participation (assesses social dimensions, such as joining in community activities, barriers and obstacles in the world surrounding the woman interviewed, and other problems, such as maintaining personal dignity). The total score ranges from 0 to 100 . A higher score is indicative of a greater limitation in daily living. ${ }^{25}$ All women should respond to the 36 questions. However, those who are unemployed or are no longer in school can only answer 32 questions, but the scores are automatically corrected for this.

The tool ASSIST, developed by WHO, provides the profile of tobacco use, alcohol and illicit substances throughout life and within the last 3 months. It consists of eight questions, and the weighted sum of answers to questions 2-7 provides a score. Following question 1, related to lifetime substance use, the second question concerned the frequency of use during the previous 3 months. Responses to this question are rated on a five-point frequency scale ranging from 'never' (in the past 3 months) to 'daily or almost daily'. This question provides critical information on the most relevant substances to the current health status of the respondent. If none of the substances has been used in the past 3 months, the interviewer can skip to the last three questions about previous problems and usage patterns in their lifetime. If any substance has been used during the past 3 months, questions 3-5 are asked, before concluding with questions 6-8. Question 3 asks about compulsive substance use in the last 3 months. This is a measure of psychological dependence. Question 4 asks about personal health, social, financial or legal problems associated with substance use occurring within the previous 3 months. Question 5 asks whether participants have failed to meet role obligations. Questions 6-8 ask about lifetime and recent problems, including whether a concern has been expressed by friends or relatives, prior attempts at controlling drug use and current or lifetime drug injection. Scores ranging from 0 to 3 indicate no need for intervention; scores from 4 to 26 indicate a brief intervention; and scores 27 or more indicate that the subject required a referral to more specific care. ${ }^{16}$

\section{Data collection and management}

Data were collected both by telephone and face-to-face interviews. On telephone conversation, contact was made by a team of interviewers from the Center for Studies in Reproductive Health of Campinas specially trained for this study. Face-to-face interviews took place in an office dedicated to this study for privacy.

All telephone interviews were recorded with the women's consent. Interview data were entered directly into a precoded online data programme developed for this purpose with Lime Survey and subsequently transferred to an SPSS package (version 20). Research assistants included the data from face-to-face interviews to electronic forms. Data collection sheets were filed appropriately and made available for access. Data consistency was rigorously checked. Initially, in the phone component of the interview, for a random sample of around $5 \%$ of women reached, a second call was performed by a research supervisor, asking the women to repeat the interview for checking consistency. Each interviewer then received feedback from the supervisor on the best way of approaching women and correcting the original file if this was the case. In the face-to-face interview, the interviewers received specific training following a specially prepared standard operations procedure. Each filled form was revised by the research team before being doubled entered in the database. For each inconsistency found, the printed form was checked again. In case of uncertainty, the woman was again contacted by telephone to confirm information.

\section{Statistical analysis}

For analysis, characteristics of women were initially compared between both groups. The instruments used were evaluated, and women from each group presenting altered scores were identified, with their respective proportions. Furthermore, the study evaluated whether different instruments identified the same women. The proportion of women and children respectively showing any altered maternal or child health instrument were assessed in both groups. For comparison of proportions of qualitative variables between the groups, $\chi^{2}$ tests are used, while the Student's t-test was used to compare quantitative variables with means $\pm \mathrm{SD}$. When applicable, risk ratios and $95 \%$ CI were also used. Then, a multivariate 
analysis was performed to identify those factors independently associated with the outcomes 'any altered maternal assessment' and 'any altered child health assessment', reporting their adjusted ORs and $95 \% \mathrm{CI}$, after controlling for possible confounding when including the predictors in the model.

\section{Ethical approval}

All women signed an informed consent form after an invitation to participate and before enrolment. For women below 18 years, the consent was also obtained from a parent or a legal representative. All procedures were performed following relevant guidelines and regulations in the country. The confidentiality about the source of information was assured.

\section{Patient and public involvement}

Patients or the public were not involved in the design, or conduct, or reporting, or dissemination plans of our study.

\section{RESULTS}

Initially, the cohort consisted of 384 eligible women with SMM and 419 without SMM. Of these 803 women, 638 $(79.5 \%)$ had face-to-face scheduled interviews, 315 from the exposed group (82.0\%) and 323 from the control group $(77.1 \%)$. Not all children of women included in the study were assessed. The evaluation was possible for 264 children in the exposure group and 307 in the control group (figure 1).

There were no differences in schooling, ethnicity, marital status, parity or time elapsed between delivery and interview in both groups. However, women in the SMM group were older and had more preterm births (table 1).

Table 2 shows that domain 4 scores of SF-36 regarding general health were significantly lower in women with SMM, while the proportion of PTSD score $\geq 50$ was similar between groups. On assessments performed during faceto-face interviews, women from the SMM group were more frequently sterile (due to any kind of surgical sterilisation or hysterectomy), had more new clinical conditions developed after the index pregnancy (including hypertension, diabetes, mental disorders, pelvic pain and so on), and a higher total score for altered functioning, while the proportions of FSFI score or women with any drug use were similar between groups. In addition, children of women with SMM were more likely to have weight (threefold) and height (1.5 fold) growth deficits and also impaired development as assessed by Denver tool (1.5 fold).

Irrespective of SMM, the proportion of women with some altered characteristics simultaneously identified by two assessment tools was relatively high (above 50\%) for WHODAS with FSFI and with PTSD, PTSD with FSFI, SF-36 with FSFI and with PTSD (table 3). The distribution of the number of altered tools per woman was relatively similar between both groups as shown in table 4 , considering only women with information gathered from all tools for maternal conditions used in the study.

There was no relationship between SMM and the presence of any altered maternal outcome. However, there was an association between maternal morbidity and any alteration in child health outcomes (table 5). While low schooling was the only factor associated with any altered maternal instrument on multivariate analysis, factors independently associated with some altered child health instruments were prematurity, a shorter elapsed time between childbirth and the interview, lower maternal schooling and higher maternal age (table 6).

\section{DISCUSSION}

The current multidimensional evaluation of several aspects of a woman's life and health following a severe maternal morbidity episode, using different instruments showed some degree of impairment associated with SMM. There was a perception of a poorer quality of life, impairment of functioning, lower reproductive capacity, development of new clinical conditions and greater impairment in growth and development of the respective children. To the best of our knowledge, this multidimensional and comprehensive assessment of the repercussions of SMM is an innovative and more complete approach to this topic to date.

In the present study, the SF-36 scores showed significant differences between groups in the domains of functional capacity, physical aspects, pain and general health, as already reported elsewhere. ${ }^{20}$ Altered PTSD had a similar prevalence between groups. ${ }^{21}$ Although the perception of worse quality of life in women with SMM, they did not report a higher proportion of PTSD. A possible explanation for the apparently conflicting data is the time elapsed between the event and the interview ranging from 6 months to 5 years. There could be a decreasing perception of PTSD, which is more strongly associated with acute or recent events. We cannot confront these data with findings in the literature, since no studies were performed similarly using both instruments.

The overall mean WHODAS score was higher in women with SMM, as well as for domains related to mobility, domestic activities and work/school activities. ${ }^{19}$ In addition, scores for the 90th percentile were similar in both groups. ${ }^{19}$ Unfortunately, these results cannot be compared with findings from other studies because this was the first time that functioning was assessed in postpartum women, at least using this instrument. Recently, however, a pilot study conducted by the WHO and using the simplified version, the WHODAS-12, applied within a broader instrument to assess morbidity (the WOICE instrument) was used in three low-income countries, both during pregnancy and in the postpartum period. The results, however, are still not available. ${ }^{26}$

WHODAS enables the understanding of health behaviour in various cultures and contributes to global public health policies. WHODAS scores are significantly 


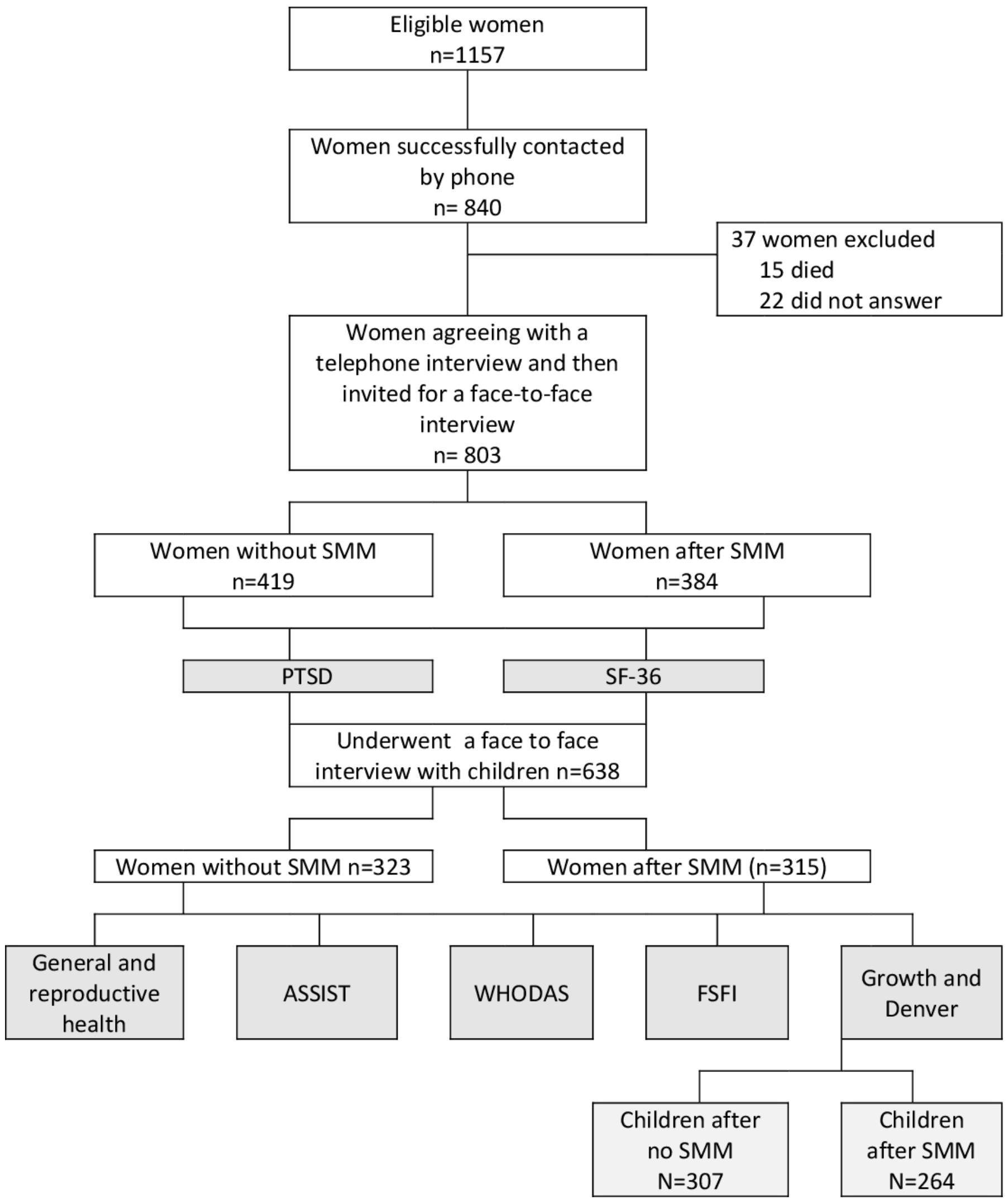

Figure 1 Flow chart of women participating in the COMMAG study. ASSIST, Alcohol, Smoking and Substance Involvement Screening Test; COMMAG, Cohort for Severe Maternal Morbidity; Denver, Denver Developmental Screening Test; FSFI, Female Sexual Function Index; growth: weight or height/age; PTSD, post-traumatic stress disorder; SF36, short form (of health form, for quality of life assessment); SMM, severe maternal morbidity=potential life-threatening condition+maternal near miss; WHODAS, WHO Disability Assessment Schedule. 
Table 1 Main sociodemographic characteristics of women included in the study according to the previous experience of SMM

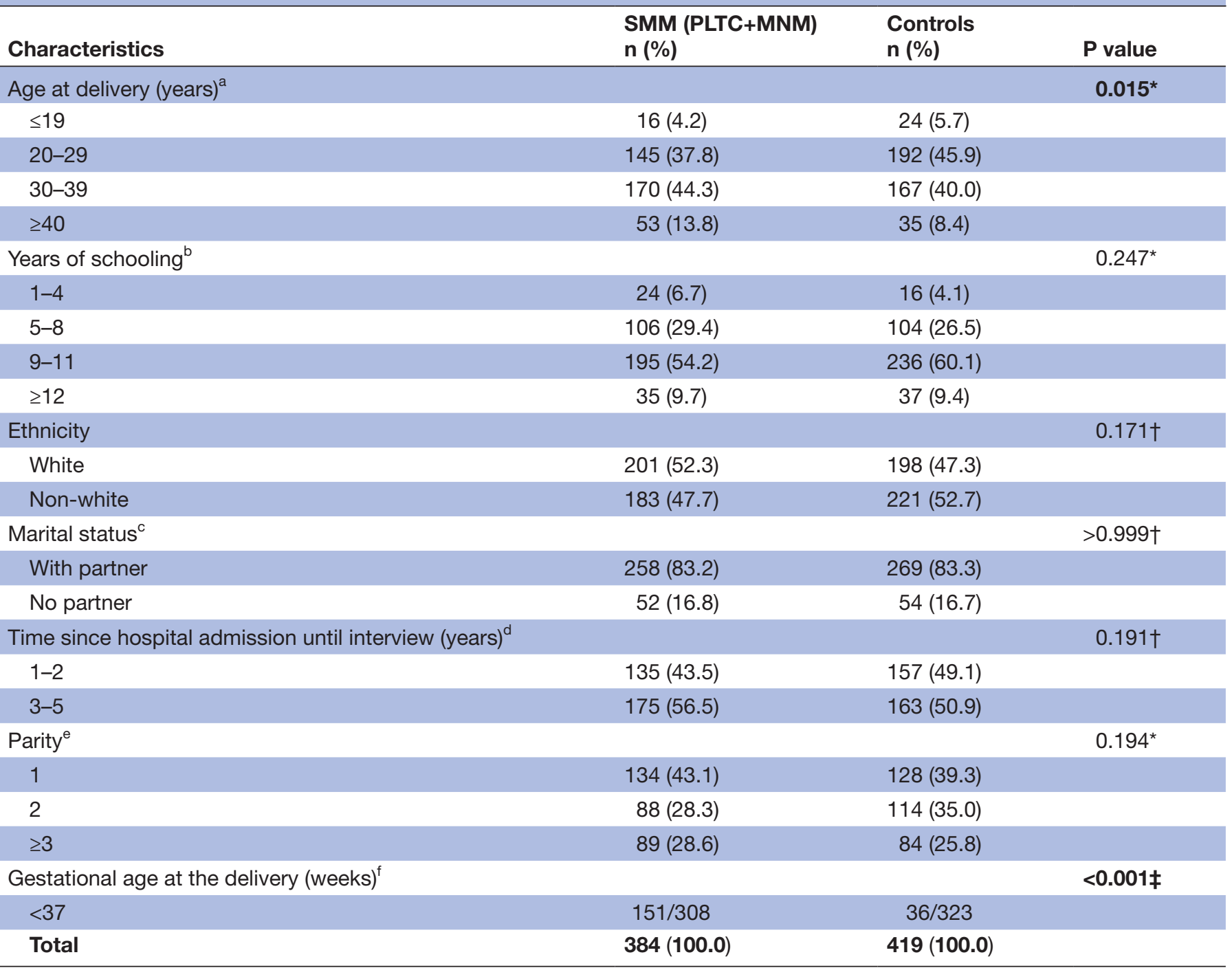

Missing information for a: 1; b: 50; c: 5; d: 8; e:1; f: 7 cases (variables c-f available only for 638 women who attended the face-to-face interview).

Controls: women without SMM.

*Pearson $\chi^{2}$ test.

†Continuity correction test.

$\ddagger$ Yates $\chi^{2}$ test.

MNM, maternal near miss; PLTC, potentially life-threatening conditions; SMM, severe maternal morbidity=potential life-threatening condition+maternal near miss.;

associated with instruments used to measure disability, such as the Medical Outcomes Study 36 - Short-Form Item and the Quality of Life instrument (WHOQOL), expanding the possibility of comparative analysis. We chose WHODAS 2.0 because of its solid theoretical foundation, excellent psychometric properties, diverse applications in different groups and settings and ease of use. For these reasons, the shorter WHODAS version was also incorporated into the new WHO tool (WOICE) to investigate the burden of associated complications and implications of pregnancy itself in the life experience and health of women. ${ }^{2627}$
There were no significant differences in the total FSFI scores when comparing groups, even for each domain. ${ }^{18}$ Women who had SMM had a worse assessment of health status and sexual function 6 months after delivery. There were no differences in the risk of postpartum depression between women with or without complications. ${ }^{10}$ An increased risk of depression and anxiety after childbirth was also described in women following severe maternal morbidity in Africa. The incidence of postpartum suicidal ideation, greater negative perception of pregnancy and/ or childbirth and even increased risk of death within 1 year postpartum for both mother and child were higher 
Table 2 Main results of the multidimensional assessment of several aspects of women's health and life according to the occurrence of severe maternal morbidity

\begin{tabular}{|c|c|c|c|c|c|}
\hline \multirow[b]{2}{*}{ Assessment } & \multicolumn{2}{|c|}{ Women after SMM } & \multicolumn{2}{|c|}{ Women without SMM } & \multirow[b]{2}{*}{ P value or $\mathbf{R R}(95 \% \mathrm{Cl})$} \\
\hline & $\mathrm{n} / \mathrm{N}$ or Mean & $\%$ or $( \pm S D)$ & $\mathrm{n} / \mathrm{N}$ or Mean & $\%$ or $( \pm S D)$ & \\
\hline \multicolumn{6}{|l|}{ Telephone interview } \\
\hline PTSD score $\geq 50$ & $112 / 382$ & 29.3 & $112 / 412$ & 27.2 & $\mathrm{p}=0.556$ \\
\hline \multicolumn{6}{|l|}{ Face-to-face interview } \\
\hline Any new clinical condition & $141 / 315$ & 44.8 & $82 / 323$ & 25.4 & $p<0.001$ \\
\hline WHODAS 2.0 score & 19.0 & $( \pm 16.2)$ & 15.8 & $( \pm 14.5)$ & $p=0.015$ \\
\hline FSFI score $<26.55$ & $131 / 282$ & 46.5 & $152 / 301$ & 50.5 & $\mathrm{p}=0.372$ \\
\hline ASSIST - any drug & $86 / 315$ & 27.3 & $79 / 323$ & 24.5 & $p=0.466$ \\
\hline \multicolumn{6}{|l|}{ Children } \\
\hline
\end{tabular}

$P$ values in bold mean they are statistically significant.

Controls: women without SMM.

ASSIST, Alcohol, Smoking and Substance Involvement Screening Test; Denver, Denver Developmental Screening Test; FSFI, Female Sexual Function Index; PTSD, post-traumatic stress disorder; RR, risk ratio; SF-36 domain 4, Short Form (of Health Form, for quality of life assessment); SMM, severe maternal morbidity=potential life-threatening condition+maternal near miss; WHODAS, WHO Disability Assessment Schedule.

for SMM. ${ }^{28} 29$ There were no significant differences between the use of tobacco, alcohol and illicit substances during pregnancy of women in both groups, nor in the period after the index pregnancy until the time of the interview. $^{22}$

We demonstrated a clear and significant growth inhibition (weight and height for age) and also impaired the development of these children. This is likely the most innovative aspect of the present study. To the best of our knowledge, medium-term and long-term growth and development of children surviving SMM conditions have yet to be evaluated. Some of these unfavourable outcomes were probably the result of the higher occurrence of prematurity among SMM cases, as suggested by the results of our multivariate analysis.

A high number of women in the group without SMM also had alterations assessed by different instruments used. SMM is the main focus. Nevertheless, it would

Table 3 Proportion of women identified with some altered characteristics simultaneously by two tools (only for those 638 women who also attended the face-to-face interview)

\begin{tabular}{|c|c|c|c|c|c|c|c|}
\hline Tools & Whodas & FSFI & PTSD & SF-36 & Assist & Growth & Denver II \\
\hline WHODAS ${ }^{a}$ & $66(100 \%)$ & 49 (17.3) & 45 (23.5) & 14 (26.9) & $16(9.7)$ & $11(10.5)$ & $9(11.7)$ \\
\hline $\mathrm{FSFI}^{\mathrm{b}}$ & $49(74.2)$ & $283(100 \%)$ & $120(62.8)$ & $29(55.8)$ & $67(40.6)$ & 47 (44.8) & $34(44.2)$ \\
\hline$S F-36^{d}$ & 14 (21.2) & $29(10.2)$ & 29 (15.2) & $52(100 \%)$ & $13(7.9)$ & $10(9.5)$ & 10 (12.9) \\
\hline ASSIST & $16(24.2)$ & $67(23.7)$ & 63 (32.9) & $13(25.0)$ & $165(100 \%)$ & $26(24.8)$ & $16(20.8)$ \\
\hline Denver II $^{f}$ & 9 (13.6) & 34 (12.1) & $28(14.7)$ & 10 (19.2) & $16(9.7)$ & 24 (22.8) & 77 (100\%) \\
\hline
\end{tabular}

Cut-offs for each instrument: WHODAS: >p90; FSFI: <26.55; PTSD: $\geq 50$; SF-36: <p10; ASSIST: any drug after index pregnancy; growth: weight or height <p10/age; Denver II: failed/impaired.

The total number of cases with altered tool score is in the shadowed boxes for each tool.

Missing information for a: 2; b:55; c:6; d:15; e:71; f: 67 cases. Proportions above 50\% are highlighted in bold.

Proportions are related to the total number of altered cases in each instrument (100\%) in columns.

ASSIST, Alcohol, Smoking and Substance Involvement Screening Test; Denver, Denver II Developmental Screening Test; FSFI, Female Sexual Function Index; PTSD, post-traumatic stress disorder; SF-36, Short Form (of Health Form, for quality of life assessment); WHODAS, WHO Disability Assessment Schedule. 
Table 4 Proportion of number of tools with altered answers for maternal conditions according to the severe maternal morbidity status

\begin{tabular}{lcc}
\hline $\begin{array}{l}\text { Number of } \\
\text { altered tools }\end{array}$ & $\begin{array}{l}\text { SMM } \\
\mathbf{n}(\%)\end{array}$ & $\begin{array}{l}\text { No severe maternal } \\
\text { morbidity } \\
\mathbf{n}(\%)\end{array}$ \\
\hline 0 & $83(30.4)$ & $89(30.0)$ \\
\hline 1 & $94(34.4)$ & $108(36.4)$ \\
2 & $52(19.0)$ & $65(21.9)$ \\
3 & $32(11.7)$ & $29(9.8)$ \\
4 & $12(4.4)$ & $4(1.3)$ \\
5 & $0(-)$ & $2(0.7)$ \\
\hline Total & $\mathbf{2 7 3 a}$ & $\mathbf{2 9 7 b}$ \\
\hline
\end{tabular}

Pearson $\chi^{2}=7.77(p=0.169)$.

Five instruments were used: WHODAS score: >p90 (41.3); FSFI score: <26,55; PTSD total score: $>50$; domain 4 of SF-36: $<\mathrm{p} 10$ (35.0); and ASSIST: any drug used after index pregnancy. Missing: $a=50, b=18$ (not all tools completed).

SMM, Severe Maternal Morbidity = Potential Life-Threatening

Condition + Maternal Near Miss.

be interesting to evaluate the subgroup without severe morbidity. These women had some morbidity, although less serious. Some recently published studies have emphasised this trend and the importance of establishing welldefined criteria for non-severe morbidity, which could contribute to the development of better public health policies and adequate allocation of resources. Ultimately, a reduction in maternal deaths could occur with interruption of the maternal morbidity spectrum and its consequences. In response to such gaps, the WHO implemented a project aimed at defining, measuring and monitoring less severe maternal morbidity, thus completing the maternal morbidity continuum. ${ }^{12}$

Considering that there are very few studies approaching medium-term and long-term repercussions of severe maternal morbidity using a multidimensional assessment, it is quite difficult to predict how generalisable

Table 5 Association of altered tools for maternal and for children's conditions with the severe maternal morbidity (SMM) status

\begin{tabular}{|c|c|c|c|c|c|}
\hline \multirow[b]{2}{*}{ Altered tools } & \multicolumn{2}{|c|}{$\begin{array}{l}\text { Women after } \\
\text { SMM }\end{array}$} & \multicolumn{2}{|c|}{$\begin{array}{l}\text { Women } \\
\text { without SMM }\end{array}$} & \multirow[b]{2}{*}{ P value* } \\
\hline & $\mathbf{n}$ & $\%$ & $\mathbf{n}$ & $\%$ & \\
\hline Any maternal $^{\mathrm{a}}$ & & & & & 0.982 \\
\hline Yes & 190 & 69.6 & 208 & $70 ; 0$ & \\
\hline No & 83 & 30.4 & 89 & 30.0 & \\
\hline Any children ${ }^{b}$ & & & & & 0.015 \\
\hline Yes & 85 & 33.9 & 70 & 23.7 & \\
\hline No & 169 & 66.1 & 225 & 76.3 & \\
\hline
\end{tabular}

Missing values for a: 68 , b:22 cases

${ }^{*}$ Continuity correction $\chi^{2}$.
Table 6 Factors independently associated with any alteration in tools for the mother and the children in multivariate analysis

\begin{tabular}{|c|c|c|}
\hline Factor & OR $(95 \% \mathrm{Cl})$ & $\begin{array}{l}\text { Adjusted } \\
\text { p value }\end{array}$ \\
\hline \multicolumn{3}{|l|}{$\begin{array}{l}\text { Any altered maternal } \\
\text { assessment }(n=560)\end{array}$} \\
\hline $\begin{array}{l}\text { Years of schooling ( } \leq 8 \\
\text { years) }\end{array}$ & $1.72(1.13$ to 2.61$)$ & 0.011 \\
\hline \multicolumn{3}{|c|}{ Any altered children assessment $(n=544)$} \\
\hline $\begin{array}{l}\text { Gestational age at birth } \\
\text { (<37 weeks) }\end{array}$ & 2.97 (1.98 to 4.48$)$ & $<0.001$ \\
\hline Age (years) & 1.05 (1.02 to 1.08$)$ & 0.003 \\
\hline Time ( $\leq 2$ years) & 1.59 (1.07 to 2.37$)$ & 0.022 \\
\hline $\begin{array}{l}\text { Years of schooling }(\leq 8 \\
\text { years) }\end{array}$ & 1.55 (1.02 to 2.36$)$ & 0.039 \\
\hline
\end{tabular}

Independent variables: group (control: 0/SMM: 1); age (years); years of schooling ( $\leq 8: 1)$; ethnicity (white: $0 /$ non-white: 1 ); marital status (no partner: 1); time since hospital admission until interview (up to 2 years: 1 ); parity ( $<2: 0 / \geq 2$ : 1 ); gestational age at the delivery ( $<37$ weeks : 1$)$; route of delivery (vaginal: $1 /$ caesarean section: 0); breast feeding (yes: 1/no: 0).

are these data for the population of women suffering the experience of a severe maternal morbidity episode. It is probable that individual, cultural and health system care characteristics could play an important role in the process on how the women's lives and that of their children are affected by such an occurrence across time.

Our study has, however, some limitations. The retrospective design of this cohort study is one of them. In addition, as a retrospective cohort, it was not possible to better assess the conditions associated with death among the apparent excess cases in the exposed group. A recent study derived from database information on multiple countries of the American continent explored more than 700000 births and found a proportion of 1 maternal death to 262 morbidities. This accounts for a morbidity prevalence of $38 \%$, with more than $50 \%$ of cases corresponding to less severe maternal morbidity, signalling the need for greater awareness about this specific group. ${ }^{30} 31$ Therefore, early identification of different morbidity spectrum, in addition to adequate and timely management of morbidity, may provide better maternal outcomes and contribute to better perinatal outcomes.

Acknowledgements This article is part of the PhD thesis of ECF under the tutorial of JGC and MLC, and presented to the Postgraduate Program on Obstetrics and Gynecology from the School of Medical Sciences, the University of Campinas in Brazil, on 21 February 2019.

Contributors Conception or design of the work: ECF, RCP, MAP and JGC; the acquisition, analysis or interpretation of data: all authors; or have drafted the work or substantively revised it: ECF, MLC and JGC; have approved the submitted version: all authors.

Funding This study was funded by the Brazilian National Research Council - CNPq (Grant 471142/2011-5) that played no role at all in the study design, implementation, data collection, analysis, interpretation of data and writing the manuscript. 
Competing interests None declared.

Patient consent for publication Not required.

Ethics approval The study was approved by the Institutional Review Board of the University of Campinas (Letter of approval 447/2009) and financed by CNPq (Grant 471142/2011-5).

Provenance and peer review Not commissioned; externally peer reviewed.

Data availability statement Data are available on reasonable request. All relevant data are within the paper, and the authors can make available materials, data and associated protocols if requested.

Open access This is an open access article distributed in accordance with the Creative Commons Attribution Non Commercial (CC BY-NC 4.0) license, which permits others to distribute, remix, adapt, build upon this work non-commercially, and license their derivative works on different terms, provided the original work is properly cited, appropriate credit is given, any changes made indicated, and the use is non-commercial. See: http://creativecommons.org/licenses/by-nc/4.0/.

ORCID iD

Jose Guilherme Cecatti http://orcid.org/0000-0003-1285-8445

\section{REFERENCES}

1 Firoz T, Chou D, von Dadelszen P, et al. Measuring maternal health: focus on maternal morbidity. Bull World Health Organ 2013;91:794-6.

2 Chou D, Tunçalp Özge, Firoz T, et al. Constructing maternal morbidity - towards a standard tool to measure and monitor maternal health beyond mortality. BMC Pregnancy Childbirth 2016;16:45.

3 Say L, Pattinson RC, Gülmezoglu AM. WHO systematic review of maternal morbidity and mortality: the prevalence of severe acute maternal morbidity (near miss). Reprod Health 2004;1:3.

4 Geller SE, Rosenberg D, Cox SM, et al. The continuum of maternal morbidity and mortality: factors associated with severity. $\mathrm{Am} \mathrm{J}$ Obstet Gynecol 2004;191:939-44.

5 Pacagnella RC, Cecatti JG, Camargo RP, et al. Rationale for a longterm evaluation of the consequences of potentially life-threatening maternal conditions and maternal "near-miss" incidents using a multidimensional approach. J Obstet Gynaecol Can 2010;32:730-8.

6 Say L, Souza JP, Pattinson RC, et al. Maternal near miss--towards a standard tool for monitoring quality of maternal health care. Best Pract Res Clin Obstet Gynaecol 2009;23:287-96.

7 Gill K, Pande R, Malhotra A. Women deliver for development. Lancet 2007;370:1347-57.

8 Filippi V, Goufodji S, Sismanidis C, et al. Effects of severe obstetric complications on women's health and infant mortality in Benin. Trop Med Int Health 2010;15:733-42.

9 Camargo RS, Pacagnella RC, Cecatti JG, et al. Subsequent reproductive outcome in women who have experienced a potentially life-threatening condition or a maternal near-miss during pregnancy. Clinics 2011;66:1367-72.

10 Waterstone M, Wolfe C, Hooper R, et al. Postnatal morbidity after childbirth and severe obstetric morbidity. BJOG 2003;110:128-33.

11 Cecatti JG, Souza JP, Parpinelli MA, et al. Brazilian network for the surveillance of maternal potentially life threatening morbidity and maternal near-miss and a multidimensional evaluation of their long term consequences. Reprod Health 2009;6:15.

12 Jenkinson C, Stewart-Brown S, Petersen S, et al. Assessment of the SF-36 version 2 in the United Kingdom. J Epidemiol Community Health 1999;53:46-50.

13 Weathers FW, Litz BT, Herman DS, et al. The PTSD checklist (PCL): reliability, validity, and diagnostic utility. paper currents at the 9th annual conference of the International Society for traumatic stress studies. San Antonio, Tx, USA, 1993.

14 Rosen R, Brown C, Heiman J, et al. The female sexual function index (FSFI): a multidimensional self-report instrument for the assessment of female sexual function. J Sex Marital Ther 2000;26:191-208.

15 World Health Organization. Measuring health and disability: manual for WHO disability assessment schedule (WHODAS 2.0. Geneva: WHO, 2010. http://whqlibdoc.who.int/publications/2010/ 9789241547598_eng.pdf

16 World Health Organization (WHO). Management of substance abuse. The assist (alcohol, smoking and substance involvement screening test) screening test version 3.0 and feedback card. Geneva: WHO, 2011. http://www.who.int/substance_abuse/activities/assist_ portuguese.pdf?ua $=1$

17 Frankenburg WK, Dodds J, Archer P, et al. The Denver II: a major revision and restandardization of the Denver developmental screening test. Pediatrics 1992;89:91-7.

18 Andreucci CB, Cecatti JG, Pacagnella RC, et al. Does severe maternal morbidity affect female sexual activity and function? Evidence from a Brazilian cohort study. PLoS One 2015; 10:e0143581

19 Silveira C, Parpinelli MA, Pacagnella RC, et al. A cohort study of functioning and disability among women after severe maternal morbidity. Int J Gynaecol Obstet 2016;134:87-92.

20 Angelini CR, Pacagnella RC, Parpinelli MA, et al. Quality of life after an episode of severe maternal morbidity: evidence from a cohort study in Brazil. Biomed Res Int 2018;2018:1-10.

21 Angelini CR, Pacagnella RC, Parpinelli MA, et al. Post-Traumatic stress disorder and severe maternal morbidity: is there an association? Clinics 2018;73:e309.

22 Pereira CM, Pacagnella RC, Parpinelli MA, et al. Drug use during pregnancy and its consequences: a nested case control study on severe maternal morbidity. Rev Bras Ginecol Obstet 2018;40:518-26.

23 Cecatti JG, Camargo RPS, Pacagnella RC, et al. ComputerAssisted telephone interviewing (cati): using the telephone for obtaining information on reproductive health. Cad Saude Publica 2011;27:1801-8.

24 Wiegel M, Meston C, Rosen R. The female sexual function index (FSFI): cross-validation and development of clinical cutoff scores. $J$ Sex Marital Ther 2005;31:1-20.

25 Ustün TB, Chatterji S, Kostanjsek N, et al. Developing the world Health organization disability assessment schedule 2.0. Bull World Health Organ 2010;88:815-23.

26 Barreix M, Barbour K, McCaw-Binns A, et al. Standardizing the measurement of maternal morbidity: pilot study results. Int $J$ Gynaecol Obstet 2018;141 Suppl 1:10-19.

27 Silveira C, Souza RT, Costa ML, et al. Validation of the who disability assessment schedule (WHODAS 2.0) 12-Item tool against the 36item version for measuring functioning and disability associated with pregnancy and history of severe maternal morbidity. Int $J$ Gynaecol Obstet 2018;141 Suppl 1:39-47.

28 Filippi V, Ganaba R, Baggaley RF, et al. Health of women after severe obstetric complications in Burkina Faso: a longitudinal study. Lancet 2007;370:1329-37.

29 Ganaba R, Marshall T, Sombié I, et al. Women's sexual health and contraceptive needs after a severe obstetric complication ("nearmiss"): a cohort study in Burkina Faso. Reprod Health 2010;7:22.

30 Serruya SJ, de Mucio B, Martinez G, et al. Exploring the concept of degrees of maternal morbidity as a tool for surveillance of maternal health in Latin American and Caribbean settings. Biomed Res Int 2017:2017:1-12.

31 Vogel JP, Souza JP, Mori R, et al. Maternal complications and perinatal mortality: findings of the world Health organization multicountry survey on maternal and newborn health. BJOG 2014;121 Suppl 1:76-88. 